\title{
METAMATERIAL STRUCTURES AND POSSIBILITY OF THEIR APPLICATION IN MICROWAVE APPLICATOR OPTIMIZATION
}

Metamaterials are special one-, two- or three-dimensional artificial structures with electromagnetic properties generally not found in nature. Due to the simultaneous negative values of permittivity $(\varepsilon)$ and permeability $(\mu)$, the wave vector and the vectors of electric-and magnetic-field intensity form a left-handed triplet with the result of antiparallel phase and group velocity and backwave propagation. These unique properties of the left-handed materials have allowed novel applications and devices to be developed. In this paper the metamaterial structures based on the split ring resonator approach for optimization of microwave applicator characteristic are used. The main field of the study is absorption of microwave-frequency field in human arm model and improvement of microwave applicator properties such a directivity and gain by means of simulations performed in CST Microwave Studio environment.

Keywords: Metamaterial structure, waveguide, microwaves, human arm model.

\section{Introduction}

Directive radiation pattern from open waveguide as a microwave applicator is strongly desirable to achieve high radiation power gain. This waveguide property is important for many technical and medical applications, which need to control the accurate electromagnetic (EM) beam focusing and maximum power gain achievement, e.g. in microwave hyperthermy therapy.

In the past few years, new methods for improving the microwave applicator gain by metamaterials were proposed [1-3] and theoretically discussed $[4,5]$. Metamaterials, which are defined as effectively homogeneous electromagnetic structures exhibiting unusual electromagnetic properties, especially the backward wave and negative refraction not readily available in nature, represent a new paradigm in electronics and photonics [6]. In those works, various metallic structures are used as metamaterials to achieve certain unusual characteristics, which are suitable for high gain design [7]. However, most of those former works are suitable for high frequency applications, such as X-band or millimeter waves.

In this paper we describe approach with implementation of metamaterial structures (MMS) with negative permeability over the microwave applicator represented by open waveguide in order to increase the gain and achieve the optimal radiation pattern in microwave X-band to improve the control of electromagnetic field focusation in microwave hyperthermy therapy.

The paper is organized as follows. The paper presents the principle of interaction of electromagnetic field with layered human body tissues and principle of proposed metamaterial structure. Next simulated results for irradiation of human arm model using conventional and tuned open waveguide are presented.

\section{Human body tissues and their interaction with electromagnetic field}

Complex relative permittivity $\left(\dot{\varepsilon}_{r}\right)$ is a parameter which is characterized by the interaction between the electric field and the bound charges in the material. Due to bound charges for lossy materials the permittivity is complex variable with both real $\left(\varepsilon_{r}^{\prime}\right)$ and imaginary $\left(\varepsilon_{r}{ }^{\prime \prime}\right)$ components

$$
\dot{\varepsilon}_{r}=\varepsilon_{r}{ }^{\prime}-j \varepsilon_{r}{ }^{\prime \prime} .
$$

Interaction between the electric field and the bound charges in tissue results in an oscillatory motion of the bound charges. The part of the motion that is $90^{\circ}$ out of phase with the electric field is characterized by the real part of the permittivity $\left(\varepsilon_{r}{ }^{\prime}\right)$ and is described as a lossless interaction. But as the bound charges oscillate, they also heat up due to friction-like forces within the molecule and from molecules nearby. This motion component is in phase with the electric field and is represented by the imaginary part of the permittivity $\left(\varepsilon_{r}^{\prime \prime}\right)$.

Thus, biological tissues are lossy materials and this loss changes the way how the wave interacts with the material and its propagation behavior. The energy will be accumulated in the lossy mater-

\footnotetext{
* Katarina Istenikova ${ }^{1}$, Dagmar Faktorova ${ }^{1}$, Adriana Savin ${ }^{2}$, Branislav Hadzima $^{3}$

${ }^{1}$ Department of Measurement and Applied Electrical Engineering, FEE, University of Zilina, Slovakia, E-mail: katarina.istenikova@fel.uniza.sk

${ }^{2}$ National Institute of Research and Development for Technical Physics, Iasi, Romania

${ }^{3}$ Department of Materials Engineering, FME, University of Zilina, Slovakia
} 
ial as wave passes through it, thus causing loss of the propagating wave energy. EM energy causes the increasing of temperature in the material [8].

\subsection{Reflection and transmission characteristics in layered body structures}

If there are several layers of different tissues, the reflection and transmission characteristics of EM waves become more complicated [9]. Due to the different permittivity of the various layers a wave impinging on the human body suffers different reflections at each boundary between two organs with different value of the permittivity, Fig.1.
L0

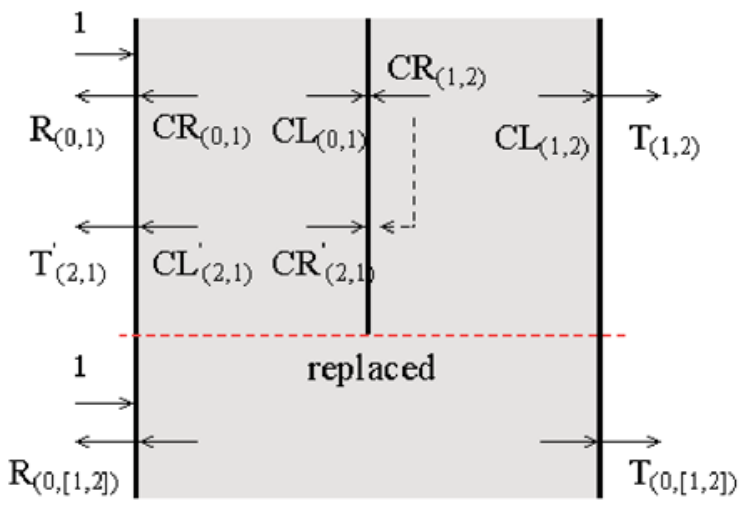

Fig. 1 Two-layered model with corresponding coefficients. $C R$ and $C L$ represents total sum of multiple reflections within sample, respectively

Hence, in the $i$-th layer, the electrical field is given by the sum of the incident field and of the reflected field from the $(i+1)$-th layer

$$
E_{i}=E_{i}^{i n c}+E_{i}^{r e f}
$$

The propagation constant in the $i$-th layer is

$$
\dot{k}=j \sqrt{\omega^{2} \dot{\varepsilon} \dot{\mu}}=\frac{j \omega}{c} \sqrt{\varepsilon_{r}^{\prime}-j \varepsilon_{r}^{\prime \prime}}
$$

and hence, the attenuation in the $i$-th layer is

$$
\alpha_{i}=\frac{\omega}{c} \operatorname{Im} \sqrt{\dot{\varepsilon}_{r}}
$$

where $\dot{\varepsilon}_{r}$ and $\dot{\mu}_{r}$ represent relative permittivity and permeability of the layer separately, $\omega$ is angular frequency, $c$ is velocity of light in vacuum [10] and for biological tissue $\mu_{r}=1$. According to that, if a classical antenna for medical purposes is matched to the air, almost all radiated field is back reflected at the air/skin interface (approximately $70 \%$ of the radiated signal). From these results it follows that proper microwave hyperthermy applicators optimized for in-body transmission are an imperative task [10].

\section{Metamaterials structure}

The currently available artificial structures are realized by using planar structures with specific topology in $x-y$ plane. These planar structures provide enhanced permeability only in the direction normal to the plane of the MMS and enhanced permittivity in the directions tangent to the plane [10]. In our paper we study the influence of negative permittivity and permeability medium on the performances of a microwave generator represented by waveguide.

Metamaterial structure used for waveguide antenna tuning consists on one side of substrate of arrays of split rectangle resonators unit cells (SRR), which were designed by Pendry et al. [10]. SRR can be described as LC resonant circuits which can be excited by a time-varying electromagnetic field with a non-negligible component applied parallel to the SRR axis. SRR structure behaves like magnetically active material in the microwave frequency range. The complex effective relative permeability of SRR structure is given by [10], [11]

$$
\dot{\mu}_{e f f}=1-\frac{f_{m p}^{2}-f_{0}^{2}}{f^{2}-f_{0}^{2}-j \gamma f},
$$

where $f$ is the frequency of microwave signal, $f_{m p}$ is the magnetic plasma frequency, $f_{0}$ is resonant frequency of SRR structure, $\gamma$ is conductivity.

On the other side of the substrate the disrupted wires array is placed, which behaves like electrically active material in the microwave frequency range. The array of parallel disrupted wires exhibits the high-pass properties for plane wave with electric vector intensity parallel to the wires. In the case when the distance between wires is smaller than the wave length of incident electromagnetic wave, the array can be taken as continuous plasma. The complex effective relative permittivity of wire structure is given by [11,12]

$$
\dot{\varepsilon}_{e f f}=1-\frac{f_{e p}^{2}}{f^{2}-j \gamma f},
$$

where $f_{e p}$ is plasma frequency, which generally depends on the geometry of wire structure like a wire radius and wire lattice constant.

Metamaterial placed in the radiation area of the open waveguide which functions as an antenna plays a role of controlling the EM wave propagation direction. In the far-field view, the sideward radiation will be reduced and forward radiation can be enhanced in the radiation patterns. As a result, a more directional and higher waveguide gain will be obtained.

The SRR structure is strongly resonant around the magnetic plasma frequency $\omega_{m p}$, which is induced by the currents and split which imitates magnetic poles. This resonant behavior is due to the capacitive element such as splits and in turn results in very high positive and negative values of permeability close to the magnetic 
plasma frequency. The SRR would yield a negative value of permeability in the case when $\omega<\omega_{m p}$. The disrupted wire structure is strongly resonant around the electric plasma frequency $\omega_{e p}$. Structure of thin wires of conductors periodically placed into dielectric substrate results a well defined plasmonic behavior also at microwave range. The wire structure yields a negative value of permittivity in the case when $\omega<\omega_{e p}[13]$.

To investigate the focusing and gain upping effect of SRR structure in this work the waveguide with metamaterial structure was tuned. The region of metamaterial structure negative permeability was proposed to embody the working frequency $12 \mathrm{GHz}$ of designed conventional waveguide WR-90.

\section{Numerical simulations}

\subsection{Model creation in CST microwave studio software}

Numerical simulations were performed with assistance of the CST Microwave Studio environment which is suitable for electromagnetic simulation of high frequency components. Metamaterial structure with double SRR with outer dimensions $5 \times 5 \mathrm{~mm}$ and thickness of the copper $35 \mu \mathrm{m}$, Fig. 2, was designated for conventional waveguide WR-90 tuning. The SRR are placed on the high frequency laminate Rogers RT/Duroid 5870 with relative permittivity $\varepsilon_{r}=2.33$ and thickness of the dielectric substrate $h=$ $=0.508 \mathrm{~mm}$ in the form of a $2-\mathrm{D}$ and provide negative reflection of phase. The number of unit resonant cells along the $x$ and $y$ axis was chosen $7 \times 7$ to fit waveguide dimensions. On the other side of the substrate the disrupted wires are placed. The incident EM wave propagates along the $\mathrm{z}$ direction, while the vector of electric intensity $E$ is oriented along the $y$ direction, and magnetic intensity $H$ is oriented along the $x$ direction.

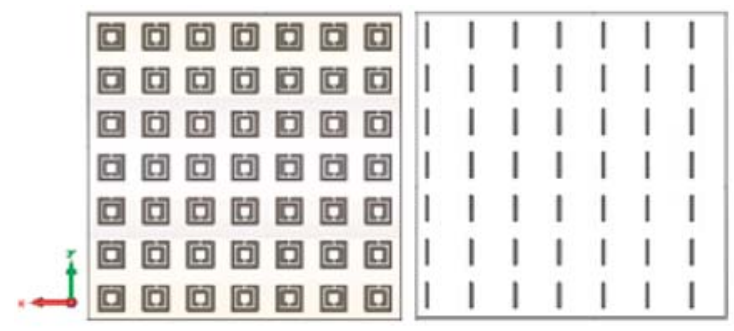

Fig. 2 Design of the metamaterial structure (front and back side)

Waveguide WR-90 working at the X-band frequency region was designed from PEC material (perfect electric conductor) with symmetry planes shown in Fig. 3; in $y z$ plane there is a magnetic symmetry and in $x z$ plane the electric symmetry. The whole simulated structure and waveguide are surrounded with vacuum.

Distance between the open waveguide and metamaterial structure influences the performance of the waveguide. Therefore this

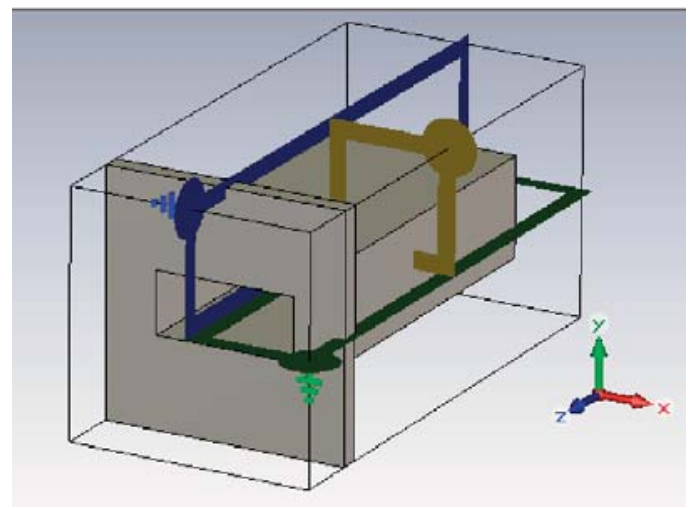

Fig. 3 Symmetry planes of the waveguide (magnetic symmetry in yz plane, electric symmetry in $x z$ plane)

parameter was numerically optimized. The simulation results in Fig. 4 have shown how this distance influences the gain of the waveguide. According to numerical results the optimal distance of metamaterial structure and conventional open waveguide was set on $18 \mathrm{~mm}$.

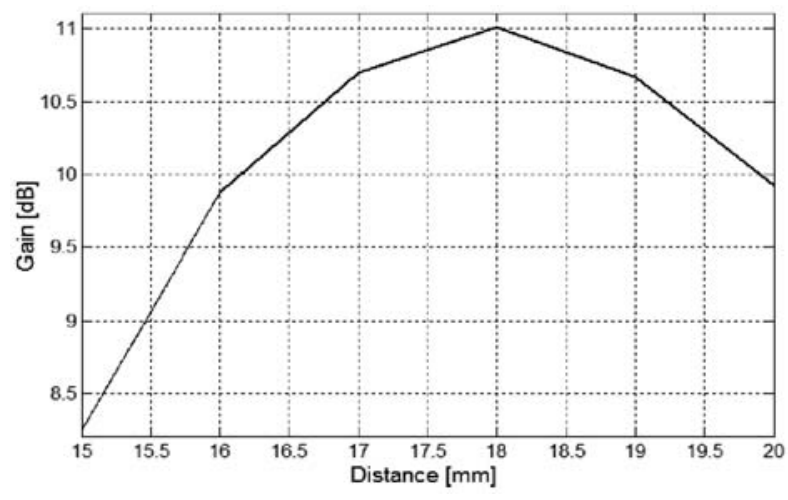

Fig. 4 Dependence between the gain of the tuned open waveguide and waveguide/metamaterial structure distance

In case that metamaterial structure is placed in $18 \mathrm{~mm}$ distance in front of the open waveguide, the gain at the frequency $12 \mathrm{GHz}$ increased from $8.23 \mathrm{~dB}$ to $10.95 \mathrm{~dB}$. According to calculation the directivity of tuned open waveguide was after metamaterial structure incorporation increased from $8.28 \mathrm{dBi}$ to $11.01 \mathrm{dBi}$.

Finally, to investigate power loss density as a main measure used in numerical dosimetry, the model of human arm consisting of four different biological structures in isothermal conditions of background was processed, Fig. 5.

Dielectric parameters used for different layers of biological sample were loaded from material library of CST Microwave Studio Suite. These data are presented in Tab. 1. 


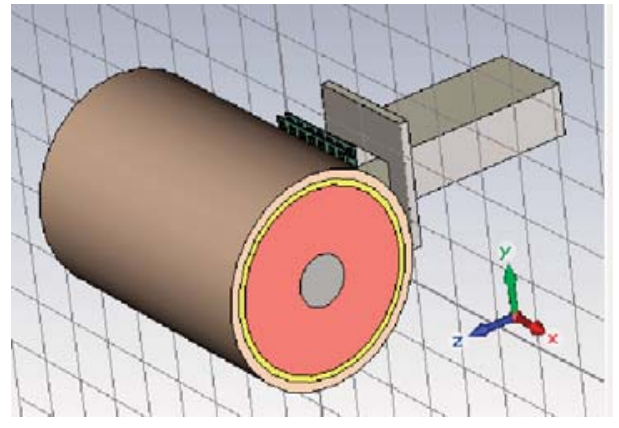

Fig. 5 Arrangement of the numerical simulations

Dielectric properties of biological layers

Table 1

\begin{tabular}{|c|c|c|c|}
\hline & $\begin{array}{c}\text { Radius } \\
(\mathrm{d} \mathrm{mm})\end{array}$ & $\begin{array}{c}\text { Permittivity } \\
\varepsilon \mathrm{r}(-)\end{array}$ & $\begin{array}{c}\text { Conductivity } \\
\sigma(\mathrm{S} / \mathrm{m})\end{array}$ \\
\hline skin & 3 & 41 & 0.7 \\
\hline fat & 2 & 5 & 0.04 \\
\hline muscle & 20 & 42.7 & 10.6 \\
\hline bone & 10 & 12 & 0.95 \\
\hline
\end{tabular}

Besides the sufficient sampling of the fields, it was very important to obtain a good approximation of the structure within the mesh. The simulated structure and the electromagnetic fields were mapped to hexagonal mesh. For the spatial discretization with a hexahedral grid, the Finite Integration Method in conjunction with the Perfect Boundary Approximation (PBA) was used, which maps the structure from the continuous world into the mesh of the discrete world. For a good compromise between the need of an accurate structure and field discretization and a short simulation time the number of meshcells was set on 10 millions.

\subsection{Results of the numerical simulations}

Power loss density closely connecting with SAR parameter was demonstrated at the frequency $12 \mathrm{GHz}$ which falls into the $\mathrm{X}$ band frequency range. The electric field vector was parallel to longitudinal axis of the models.

Power loss density value for presented human arm model at frequency $12 \mathrm{GHz}$ is shown in Fig. 6. Local power loss density was given as a numerical value per volume element and becomes a space distribution function.

In the first case the human arm model by waveguide WR 90 was irradiated. The maximum value of the power loss density reaches $11477 \mathrm{~W} / \mathrm{m} 3$ and was scattered in the muscle layer in direction radiation pattern from the waveguide. In the second simulation the suggested metamaterial structure between the open waveguide and human arm model was placed. As was shown in the color range, metamaterial structure causes increasing value of power loss density
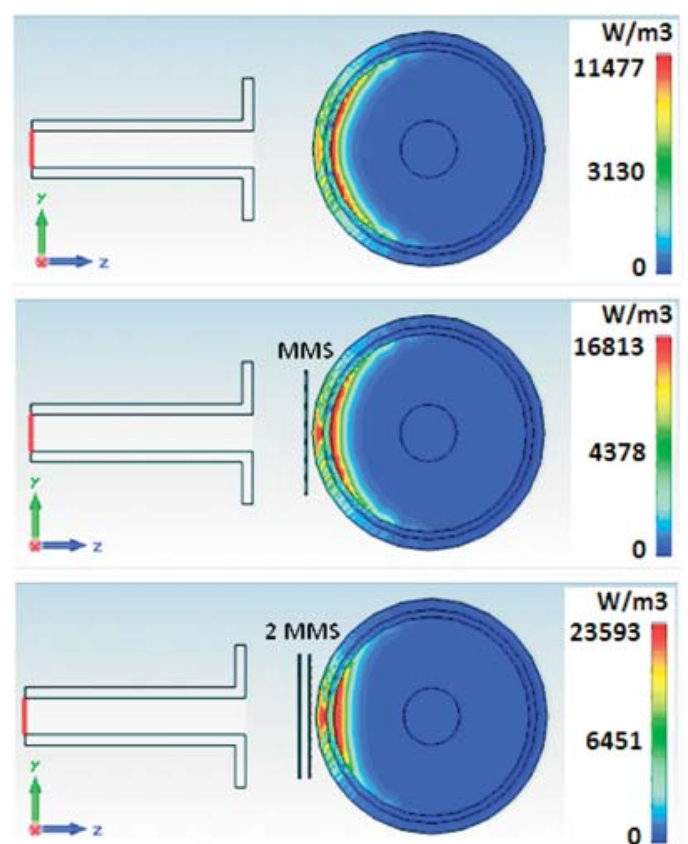

Fig. 6 Influence of the number of metamaterial structures (MMS) on the power loss density in the human arm model

in irradiated area of the model in comparison with non-tuned open waveguide case from 11477 to $16813 \mathrm{~W} / \mathrm{m} 3$. In the third simulation the additional metamaterial structure was added between the open waveguide and model, so that optimal distance $18 \mathrm{~mm}$ between the structure and waveguide was hold. The additional metamaterial structure caused subsequent increasing of the directivity which led into the doubled focusation of electromagnetic energy (23593 $\mathrm{W} / \mathrm{m} 3$ ) in comparison with non-tuned open waveguide case and the electromagnetic energy was much more concentrated in the impact area of the metamaterial structure.

However, in Fig. 6 it can be seen that used EM field in the fat layer created subdermal hot spots which are complicated to treat and thus, must be avoided. A common way of mitigating this effect is to place a layer of water between the microwave source and the irradiated part of human body.

\section{Conclusions}

The experimental and numerical results connected with the study of dielectric properties of biological materials and, on the other hand the influence of dielectric properties of biological materials on electromagnetic wave propagation gives us the information very useful in medical therapy using microwaves.

In this work the appropriate metamaterial structure was suggested by using CST Microwave Studio software for operating waveguide frequency in the negative region metamaterial structure permeability. The numerical simulations were used at the designing of both, microwave applicator and metamaterial structure. 
Also for the calculation of microwave applicator parameters such gain and directivity with numerical simulation using commercial software was done.

The simulation results showed that appropriate design of metamaterial structure improves the constitutive properties of microwave applicator - gain and directivity. The presented numerical results are useful at the microwave applicator parameters optimization used for microwave hyperthermia at malignant tumors treatment. The obtained numerical results can be also useful at microwave tomography of chosen human body parts.

\section{Acknowledgements}

This work has been supported from Slovak Research and development agency APVV Bilateral project SK-RO-0015-10 and Tatra banka grand program "Studenti do sveta" as the project 11sds046. The authors are grateful for the support of experimental works by project VEGA No. 1/0100/11 and No. 1/0743/12.

The author would like to thank Technische Universitat Ilmenau in Germany for support of this work by international program IPID - International Promovieren in Deutschland.

\section{References}

[1] TAJEH, G. et al.: Compact Directive Antennas Using Metamaterials. $12^{\text {th }}$ Intern. Symposium on Antennas, 2002, pp. 101-104.

[2] LEI, Z. et al.: Directive Emissions from Subwavelenght Metamaterial - Based Cavities. Appl. Phys. Lett, 2005, vol. 53, pp. 1-3.

[3] ALICI, K. B. F. et al.: Optimization and Tenability of Deep Subwavelenght Resonators for Metamaterial Applications: Complete Enhanced Transmission through a Subwavelenght Aperture. Opt. Express, 2009, vol. 17, pp. 5933-5943.

[4] ALU, A. ET AL.: Metamaterials Covers Over a Small Aperture. IEEE Trans. on Antenna and Propagation, 2006, vol. 54, pp. 16321642.

[5] FAKTOROVA, D.: Mater. Eng. - Mater. inz. 16, 2009, 9-13.

[6] BAENA, J. D., BONACHE, J., MARTIN, F. et al.: Equivalent-Circuit Models for Split-Ring Resonators and Complementary Split Ring Resonators Coupled to Planar Transmission Lines. IEEE Transactions on Microwave Theory and Techniques, 2005, vol. 53, pp. 1451-1461.

[7] LAI, A., CALOZ, C., ITOH, T.: Composite Right/Left-Handed Transmission Line Metamaterials. IEEE Microwave Magazine, 2004, vol. 5, pp. 34-50.

[8] PEYMAN, A.: Dielectric Properties of Tissues, Variation with Structure and Composition. Proc. of Conference ICAA, 2004, pp. 863-864.

[9] KOZLIKOVA, K., MARTINKA, J.: Zaklady spracovania biomedicinskych merani - cast II. [Basics of Biomedical Measurements Processing - part II.]. ASKLEPIOS, Bratislava 2009, ISBN 978-80-7167-137-4, 202 p.

[10] PANCERA, E., BARBA, H., LI, X., JALILVAND, M., ZWICK, T.: UWB Antennas Optimization for In-Body Radiation. Proc. of the 6th German Microwave Conference, 2011.

[11] PENDRY, J. B.: Magnetism Covers Over a Small Aperture. IEEE Trans. Microwave Theor. Tech., 1999, vol. 47, pp. $2075-2084$.

[12] LIU, Y. H., ZHAO, X. P.: Investigation of Anisotropic Negative Permeability Medium Cover for Patch Antenna. IET Microwave, Antennas and Propagation, 2008, vol. 2, pp. 737-744.

[13] FAKTOROVA, D., OMELKA, P., ISTENIKOVA, K.: Antenna Parameters Enhancement by Using Artificial Magnetic Structure. J. of Electrical Engineering, 2010, vol. 61, pp. 156-159. 